Pesq. Vet. Bras. 35(10):844-852, outubro 2015

DOI: $10.1590 / \mathrm{S} 0100-736 \mathrm{X} 2015001000005$

\title{
Meningiomas em cães: aspectos clínicos, histopatológicos e imuno-histoquímicos ${ }^{1}$
}

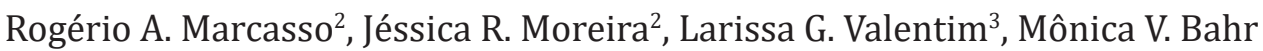 \\ Arias $^{3}$ e Ana Paula F.R.L. Bracarense ${ }^{2 *}$
}

\begin{abstract}
Marcasso R.A., Moreira J.R., Valentim L.G., Arias M.V.B. \& Bracarense A.P.F.R.L. 2015. [Meningiomas in dogs: clinical, histopathological and immunohistochemical aspects.] Meningiomas em cães: aspectos clínicos, histopatológicos e imuno-histoquímicos. Pesquisa Veterinária Brasileira 35(10):844-852. Laboratório de Patologia Animal, Departamento de Medicina Veterinária Preventiva, Universidade Estadual de Londrina, Rodovia Celso Garcia Cid Km 380, Londrina, PR 86057-970, Brazil. E-mail: ana.bracarense@pq.cnpq.br

Neoplasias of the central nervous system (CNS) of small animals are frequently diagnosed; however, data relative to prevalence are scarce. The aim of this study was to evaluate retrospectively the occurrence of primary CNS tumors in dogs in a Veterinary Teaching Hospital and describe clinical, histopathological and immunohistochemical aspects of the most common tumors. Fourteen cases of CNS primary neoplasia (prevalence of $0.27 \%$ ) were identified from 1998 to 2013; from these 11 were diagnosed as meningiomas. The mean age of dogs with meningiomas was 10 years; male (7/11) and the Boxer breed (3/11) were most commonly represented. Seven meningiomas were located in the spinal cord and four intracranially; the main clinical signs were impaired locomotion and seizures, respectively. Pulmonary metastasis was found in two cases. Myelography was performed in six dogs with spinal meningiomas. In these cases it was effective in demonstrating the deviation or interruption of the contrast column. Exploratory surgery to confirm diagnosis or remove the tumor was performed in five animals and the survival rate varies 85 to 960 days. Meningiomas were histologically classified as transitional (4/11), meningothelial $(2 / 11)$, papillary (2/11), angiomatous (1/11), microcystic (1/11) and anaplastic (1/11). Evaluation of histological grade was performed; 10 meningiomas were classified as grade I and one as grade III. The neoplastic cells of eight tumors (8/11) were positive for Masson's trichrome and one with the Congo red histochemical techniques. The immunohistochemical assays revealed in all tumors a positive immunoreactivity for vimentin but negative staining for factor VIII and p53. Immunolabelling for S100 (6/11), GFAP (5/11) and pancytokeratin (3/11) showed a variable staining intensity. The mean cell proliferation index was 3.2 mitotic figures and 3.4\% for Ki-67 immunostaining. The results confirmed that meningiomas are the most frequent primary CNS neoplasia in dogs, with variation in histological subtypes. Histochemical and immunohistochemical characterization contributed to the diagnosis; however, studies of the expression of other genes are necessary to assist with the prognosis of this kind of neoplasia.
\end{abstract}

INDEX TERMS: Neoplasms, meningiomas, central nervous system, immunohistochemistry, dogs.

\footnotetext{
${ }^{1}$ Recebido em 5 de dezembro de 2014.

Aceito para publicação em 28 de julho de 2015.

${ }^{2}$ Laboratório de Patologia Animal, Departamento de Medicina Veterinária Preventiva, Universidade Estadual de Londrina (UEL), Rodovia Celso Garcia Cid Km 380, Londrina, PR 86057-970, Brasil. *Autor para correspondência: ana.bracarense@pq.cnpq.br

${ }^{3}$ Departamento de Clínicas Veterinárias, UEL, Cx. Postal 10.011, Londrina, PR 86057-990.
}

RESUMO.- As neoplasias no sistema nervoso central (SNC) de animais de companhia são frequentemente diagnosticadas, no entanto dados sobre prevalência são escassos. 0 objetivo deste estudo foi avaliar retrospectivamente a ocorrência de neoplasias primárias de SNC em cães atendidos em um Hospital-Escola Veterinário e descrever aspectos clínicos, histopatológicos e imuno-histoquímicos dos 
tumores mais frequentes. Quatorze casos (prevalência de $0,27 \%$ ) de neoplasias primárias de SNC foram identificados no período de 1998 a 2013 e destes, 11 tiveram o diagnóstico de meningiomas. A idade média dos animais com meningioma foi 10 anos, sendo machos (7/11) e a raça Boxer (3/11) os mais afetados. Sete meningiomas eram espinhais e quatro intracranianos, sendo os principais sinais clínicos alteração na locomoção e convulsões, respectivamente. Metástase pulmonar ocorreu em dois casos. Em seis animais com meningioma espinhal foi realizada a mielografia, sendo que em um também foi realizada a tomografia. Em todos os casos os exames foram efetivos na visualização de desvio ou interrupção da coluna de contraste, com alterações sugestivas da presença de massa. Em cinco animais realizou-se cirurgia exploratória visando a confirmação da suspeita clínica ou retirada da massa, sendo que a sobrevida variou de 85 a 960 dias. Na avaliação histopatológica, os meningiomas foram classificados em transicional (4/11), meningotelial (2/11), papilar (2/11), angiomatoso (1/11), microcístico (1/11) e anaplásico (1/11). Destes, oito (8/11) apresentaram marcação positiva para tricrômio de Masson e um para vermelho congo nas técnicas histoquímicas. No painel imuno-histoquímico, todos os casos apresentaram imunomarcação positiva para vimentina, mas imunomarcação negativa para fator VIII e p53. A imunomarcação para S100 (6/11), GFAP (5/11) e pancitoqueratina (3/11) foi de intensidade variável. Na graduação histológica, dez meningiomas eram grau I e um grau III. 0 índice médio de proliferação celular foi de 3,2 figuras de mitose e 3,4\% avaliando a expressão de Ki-67. Os resultados confirmaram que os meningiomas são a neoplasia primária de SNC mais prevalente em cães, com variação nos subtipos histológicos. A caracterização histoquímica e imuno-histoquímica contribuiu com a determinação do diagnóstico, no entanto, estudos envolvendo a expressão de outros genes são necessários para auxiliar no prognóstico dessas neoplasias.

TERMOS DE INDEXAÇÃO: Neoplasias, meningiomas, sistema nervoso central, imuno-histoquímica, cães.

\section{INTRODUÇÃO}

A ocorrência de neoplasias primárias no sistema nervoso central (SNC) de cães apresenta uma incidência crescente em decorrência do aumento da expectativa de vida somado ao avanço dos métodos de diagnóstico por imagem, como a ressonância magnética e a tomografia computadorizada (Koestner \& Higgins 2002).

O diagnóstico de neoplasias primárias do sistema nervoso é frequente em cães e gatos (Koestner \& Higgins 2002). A ocorrência de neoplasias intracranianas é estimada em 14,5 cães para cada 100.000 indivíduos, no entanto não há estimativas em relação a neoplasias da medula espinhal ou dos nervos periféricos (Fernández \& Bernardini 2010). A incidência de neoplasias encefálicas é maior em cães braquicefálicos, principalmente gliomas nas raças Boxer e Boston terrier (Frankhauser et al. 1974). Em gatos não há predisposição racial relatada (LeCouteur \& Withrow 2007).

As neoplasias no SNC têm maior ocorrência em animais adultos a idosos, no entanto, esporadicamente acometem animais jovens, com três anos ou menos (Koestner \& Higgins 2002). Não há predisposição sexual em animais (Cordy 1978), entretanto em humanos, homens apresentam maior incidência de neoplasias primárias no SNC (Surawicz et al. 1999).

Em cães e gatos, os mecanismos envolvidos no desenvolvimento de neoplasias não estão totalmente esclarecidos, mas a dieta, o ambiente, o trauma craniano e fatores genéticos, imunológicos, químicos e virais podem predispor a sua ocorrência (LeCouteur \& Withrow 2007). Em seres humanos, esses mesmos fatores e também a exposição a cigarro, álcool, ionização ou radiação, habitação próxima a indústrias e regiões metropolitanas e polimorfismo de genes relacionados ao metabolismo carcinogênico, oxidativo e do reparo de DNA têm sido considerados como fatores de risco (Wrensch et al. 2002). Dados sobre causas de neoplasias cerebrais são escassos na literatura. No homem observou-se que em meningiomas frequentemente ocorre inativação do gene de supressão tumoral Merlim, resultando em falhas no controle do crescimento e motilidade celular (Pavelin et al. 2014).

Devido ao seu caráter crônico, as neoplasias causam sinais neurológicos progressivos (Summers et al. 1995). Alterações intracranianas como edema, hidrocefalia e hemorragia podem ocorrer secundariamente (Kraft \& Gavin 1999, Stummer 2007). Convulsão é o sinal neurológico mais comum em cães e gatos (Snyder et al. 2006, Tomek et al. 2006), mas recentemente relatou-se a alteração de consciência como o sinal clínico de maior ocorrência (Santos et al. 2012). Outros sinais podem acompanhar esta afecção, como síndrome vestibular, andar em círculos, cegueira, dor em região cervical, anisocoria, tremores de cabeça e regurgitação (Snyder et al. 2006).

Dentre as técnicas de diagnóstico por imagem, a ressonância magnética e a tomografia computadorizada permitem a análise do parênquima encefálico e medular, porém não possibilitam a distinção do tipo do tumor devido a padrões muito semelhantes apresentados entre as neoplasias (Kraft et al. 1997, Kraft \& Gavin 1999). Alguns meningiomas espinhais na ressonância magnética apresentam padrão de captação de contraste bastante sugestivo (Petersen et al. 2008). Meningiomas císticos com localização ventricular apresentam aspectos de imagem semelhantes ao ependimoma, papiloma de plexo coroide e neurocitoma (Qiu et al. 2014). Além disso, outras neoplasias podem ter características macroscópicas e de localização semelhantes (melanoma espinhal mimetizando meningioma) (Li et al. 2014). Portanto, o diagnóstico definitivo é estabelecido por meio de exame histopatológico e classificação histológica. No caso de neoplasias espinhais, a mielografia permite observar se há desvio da coluna de contraste no espaço subaracnoide, permitindo detectar expansão intramedular ou compressão medular, possibilitando a inclusão da neoplasia nos diagnósticos diferenciais de cães com síndromes medulares (Bagley 2010).

A abordagem cirúrgica possibilita a realização de biopsias ou a completa remoção da neoplasia (Tomek et al. 2006, Bagley 2010). A cirurgia é um dos principais tratamentos realizados em neoplasias do SNC. 0 objetivo é a completa 
ressecção da massa para diminuir a compressão do tecido nervoso. Glicocorticoides podem auxiliar no tratamento inicial, pois alteram a permeabilidade da barreira hematoencefálica e reduzem o edema peritumoral (Koestner \& Higgins 2002). As neoplasias medulares, por apresentarem localização mais acessível, têm apresentado incremento na sobrevida dos animais, principalmente quando se opta por cirurgia associada a radioterapia e quimioterapia (Bagley 2010).

0 tempo de sobrevida em humanos é estimado em 2 a 5 anos, variando de acordo com o grau histológico da neoplasia e a idade do paciente (Davis et al. 1999). Em cães não se observou correlação entre o grau do tumor, os achados de imagem e a sobrevida do animal (Petersen et al. 2008, Jose-Lopez et al. 2013).

A classificação histológica das neoplasias do SNC em cães e gatos considera principalmente o tipo celular, o comportamento e o padrão topográfico (Koestner \& Higgins 2002). Em relação aos meningiomas, os subtipos histológicos utilizados para a classificação em animais e no homem são os mesmos: meningotelial, fibroblástico, transicional, psamomatoso, papilar, microcístico, mixoide, angiomatoso e anaplásico (Koestner \& Higgins 2002). Acúmulos extracelulares e perivasculares de amiloide raramente são observados nos meningiomas caninos (Schoniger et al. 2013).

As características imuno-histoquímicas dos meningiomas caninos são similares às descritas em humanos. Há marcação forte e difusa para o filamento intermediário vimentina na maioria das células neoplásicas (Barnhart et al. 2002). A marcação para citoqueratina é variável e frequentemente focal. A expressão da proteína glial fibrilar ácida (GFAP) raramente é positiva, enquanto que a marcação para as proteínas S100, sinaptofisina e enolase neurônio específica geralmente é negativa (Barnhart et al. 2002).

Marcadores genéticos e moleculares podem auxiliar no estabelecimento do prognóstico. Marcadores de proliferação celular, como a proteína Ki-67, são utilizados para determinar a fração de crescimento da neoplasia. A quantificação da expressão desta proteína permite estimar o grau de malignidade e correlacionar com o prognóstico (Scholzen \& Gerdes 2000). A expressão da proteína p53, responsável pela regulação do ciclo celular e apoptose, também é utilizada como fator prognóstico (Schmitt 1999). Recentemente verificou-se que meningiomas humanos com maior expressão de Ki-67 e p53 estavam associados à inativação do gene Merlim (Pavelin et al. 2014). Em cães, constatou-se que, utilizando o anticorpo anti-Ki-67, o índice proliferativo médio nos meningiomas foi de 5,7\%. Não ocorreu mar- cação para a proteína p53 nas neoplasias primárias de SNC (Violin 2009).

Não há um painel histoquímico e imuno-histoquímico estabelecido para o diagnóstico de meningiomas em cães. Além disso, a maioria dos estudos sobre prevalência, sobrevida e classificação de meningiomas apresenta dados de casuística internacional ou de amostras humanas. Assim, o objetivo deste estudo retrospectivo foi reunir cães com neoplasias primárias de SNC atendidos em um Hospital-Escola Veterinário em um período de 15 anos e avaliar aspectos epidemiológicos, clínicos, histopatológicos, histoquímicos de casos diagnosticados como meningioma, além de elaborar painel imuno-histoquímico, contribuindo para o entendimento da patogenia e para diagnóstico dos meningiomas.

\section{MATERIAL E MÉTODOS}

Realizou-se estudo retrospectivo dos registros de neoplasias na espécie canina do Laboratório de Patologia Animal da Universidade Estadual de Londrina do período de 1998 a 2013. Dentre os diagnósticos de neoplasias primárias do SNC selecionaram-se os diagnósticos de meningioma.

Posteriormente foram avaliados aspectos epidemiológicos como raça, idade, sexo, localização do tumor, sinais clínicos e tempo de evolução. Os métodos de diagnóstico e tratamento, quando disponíveis também foram avaliados.

As amostras selecionadas apresentavam-se em blocos parafinizados de onde foram obtidos cortes de $5 \mu \mathrm{m}$ de espessura. Os cortes foram submetidos às colorações de hematoxilina e eosina (HE), tricrômio de Masson (TM) e vermelho Congo para avaliação histopatológica e histoquímica e classificação das neoplasias. A classificação histológica foi realizada de acordo com Koestner \& Higgins (2002). A determinação do grau tumoral foi feita de acordo com Louis et al. (2007) que consideram meningiomas como grau I, meningioma atípico como grau II e meningioma anaplásico grau III. Fez-se a avaliação como presença ou ausência de marcação no TM, considerando positivo quando havia áreas coradas em azul entre as células neoplásicas. A avaliação de depósitos de amiloide foi realizada em microscopia de luz polarizada, sendo considerado positivo quando se observava refringência amareloesverdeada.

Para a avaliação imuno-histoquímica os cortes histológicos em lâminas previamente silanizadas foram desparafinizados e submetidos à recuperação antigênica pelo calor, usando forno de micro-ondas durante 13 minutos. As soluções utilizadas para a recuperação antigênica e as diluições dos anticorpos primários estão dispostas no Quadro 1. Posteriormente seguiu-se o bloqueio da peroxidase endógena e incubação com os anticorpos primários em câmara úmida refrigerada por 18 horas a $4^{\circ} \mathrm{C}$. Em seguida, procedeu-se a incubação com anticorpo secundário pelo método do polímero (Invitrogen ${ }^{\mathrm{TM}}$, Camarillo, CA) durante 20 minutos em temperatura ambiente e revelação com a solução cromógena de

\section{Quadro 1. Anticorpos, recuperação antigênica, diluição, clone e controle} utilizados na imuno-histoquímica nos 11 casos de meningioma canino

\begin{tabular}{lcccc}
\hline \multicolumn{1}{c}{ Anticorpos } & Clone & Diluição & $\begin{array}{c}\text { Recuperação antigênica } \\
\text { (micro-ondas) }\end{array}$ & Controles \\
\hline Vimentina & V9 & $1: 100$ & Tris-EDTA pH 9 & Fibrossarcoma \\
S100 & Policlonal & $1: 100$ & Tris-EDTA pH 9 & Córtex cerebral \\
Fator VIII & Policlonal & $1: 100$ & Tris-EDTA pH 9 & Pulmão \\
Pancitoqueratina & AE1/AE3+5D3 & $1: 100$ & Citrato pH 6 & Intestino \\
GFAP & Policlonal & $1: 100$ & Tris-EDTA pH 9 & Córtex cerebral \\
Ki-67 & 7B11 & $1: 50$ & Tris-EDTA pH 9 & Fibrossarcoma \\
P53 & BP53.12 & $1: 50$ & Tris-EDTA pH 9 & Carcinoma mamário
\end{tabular}


diaminobenzidina (Invitrogen ${ }^{\mathrm{TM}}$, Frederick, MD). Seguiu-se a contra-coloração com hematoxilina de Harris e montagem da lâmina com resina comercial. Controles positivos e negativos de cães foram utilizados em todas as reações de acordo com as recomendações do fabricante.

Avaliou-se a expressão como positiva ou negativa para os anticorpos: vimentina, pancitoqueratina, proteína S100, GFAP e fator VIII. A avaliação da imunoexpressão de p53 e Ki-67 foi realizada por meio da contagem de 500 células. A marcação foi considerada positiva quando o núcleo celular apresentava-se castanho. 0 percentual de proliferação celular foi obtido por meio da fórmula: IP= número de células imunoreagentes x 100/ 500 células.

\section{RESULTADOS E DISCUSSÃO}

Em 15 anos (1998-2013) foram diagnosticados 5083 casos de neoplasias em cães. Os diagnósticos foram estabelecidos por meio de citologia, histopatologia de biópsia incisional ou excisional e de fragmentos teciduais colhidos em necropsias. Destes casos, 15 cães $(0,29 \%)$ tiveram o diagnóstico histopatológico de neoplasia primária de SNC. Onze cães com diagnóstico de meningioma no SNC foram incluídos neste estudo. No Brasil não há estudos com relação à prevalência, mas a literatura internacional referencia uma ocorrência de 14,5 a cada 100.000 cães (Fernández \& Bernardini 2010).

Neste estudo, os meningiomas foram as neoplasias primárias de maior ocorrência $(11 / 15)$, resultado semelhante ao da literatura (Kraft et al. 1997, Snyder et al. 2006, Santos et al. 2012). As demais neoplasias diagnosticadas foram ependimoma papilar (1), astrocitoma protoplasmático (1), astrocitoma anaplásico (1) e papiloma do plexo coroide (1).

Avaliando os casos de meningioma, com relação ao sexo, $63,6 \%$ dos cães eram machos e 36,4\% fêmeas, o que difere dos dados da literatura veterinária, que relatam maior prevalência em fêmeas (Adamo et al. 2003, Platt et al. 2006). 0 efeito dos hormônios femininos na ocorrência de meningiomas em cães e gatos não é claro, portanto a esterilização cirúrgica precoce realizada em alguns países nos animais de companhia dificulta essa investigação (Adamo et al. 2003). Porém, não se verificou indiretamente tais efeitos, visto que a maioria dos animais não é castrada precocemente no Brasil e neste estudo houve maior acometimento de machos. Em seres humanos demonstrou-se que mulhe- res portadoras de meningiomas apresentam maior número de receptores para progesterona (Hsu et al. 1997).

Em relação às raças observou-se que três cães eram da raça Boxer, dois sem raça definida e dois Poodle (Quadro 2). A maior frequência da raça Boxer é similar à relatada em outros estudos sobre meningiomas em cães (Snyder et al. 2006, Santos et al. 2012). A idade média dos animais afetados foi de 10 anos, variando entre sete e 13 anos, similar ao referido na literatura (Koestner \& Higgins 2002, Petersen et al. 2008).

Dos 11 meningiomas, sete eram espinhais e quatro encefálicos. Com relação à localização no canal vertebral, constataram-se duas neoplasias na região cervical, duas na torácica e três na lombar (Quadro 2). Esse dado difere da literatura que refere os meningiomas intracranianos como mais frequentes em cães (Patnaik et al. 1986). Os meningiomas espinhais em cães se localizam com maior frequência nas regiões cervical e lombar, enquanto que em humanos a localização torácica é mais comum (Petersen et al. 2008, Jose-Lopez et al. 2013). Em cães os meningiomas nas vértebras torácicas são incomuns (Bagley 2010).

Dos 11 animais, dois (um meningioma transicional e um papilar) apresentaram metástases pulmonares e renais. A ocorrência de metástases não é comum em meningiomas, podendo ocorrer por via hematógena ou pelo líquido cerebroespinhal (Long 2006). A baixa incidência de metástases pode estar relacionada à presença de intercomunicações desmossomais e interdigitações entre as células das meninges, que reduzem a esfoliação celular (Schulman et al. 1992). Em um relato de caso de meningioma em uma mulher, observou-se a ocorrência de metástases em pulmão, fígado, mediastino, osso e coração (Yu et al. 2014). Em cães há um relato de três meningiomas meningoteliais com metástases pulmonares (Schulman et al. 1992). Apesar de raros, deve se fazer um controle de recidivas e pesquisa de metástases pulmonares e nos demais órgãos no acompanhamento clínico desses pacientes. Não há protocolo terapêutico definido para os animais com metástases de meningiomas.

Os principais sinais clínicos evidenciados nos meningiomas encefálicos foram convulsão e andar compulsivo. Sinais como ataxia, torção de cabeça e andar em círculos

Quadro 2. Informações de resenha, localização, sobrevida, classificação, painel histoquímico, imuno-histoquímico e proliferação celular de 11 casos de meningioma canino

\begin{tabular}{|c|c|c|c|c|c|c|c|c|c|c|c|c|c|c|c|c|}
\hline \multirow[t]{2}{*}{ Raça } & \multirow[t]{2}{*}{ Sexo } & \multirow{2}{*}{$\begin{array}{l}\text { Idade } \\
\text { (anos) }\end{array}$} & \multirow[t]{2}{*}{ Local } & \multirow[t]{2}{*}{ Sobrevida } & \multirow{2}{*}{$\begin{array}{c}\text { Classificação } \\
\text { histopatológica }\end{array}$} & \multirow{2}{*}{$\begin{array}{l}\text { Grau do } \\
\text { tumor }\end{array}$} & \multirow{2}{*}{$\begin{array}{c}\text { Histoquímica } \\
\text { Masson }\end{array}$} & \multicolumn{9}{|c|}{ Painel Imuno-histoquímico } \\
\hline & & & & & & & & VC & $\mathrm{Vi}$ & PCK & S100 & GFAP & FVIII & P53 & Ki-67 & Mit \\
\hline Boxer & M & 9 & $\mathrm{C} 3-\mathrm{C} 5$ & eutanásia & angiomatoso & I & - & - & + & - & + & + & - & - & $2,6 \%$ & 2 \\
\hline SRD & M & 12 & L4-L5 & eutanásia & anaplásico & III & + & - & + & $(+)$ & + & $(+)$ & - & - & $3,4 \%$ & 9 \\
\hline Dálmata & M & 9 & T2-T4 & 960 dias & transicional & I & - & - & + & - & + & - & - & - & $7,0 \%$ & 6 \\
\hline Boxer & $\mathrm{F}$ & 8 & T13-L2 & eutanásia & meningotelial & I & + & - & + & - & + & + & - & - & $7,4 \%$ & 0 \\
\hline Poodle & M & 9 & T2-T3 & 85 dias & meningotelial & I & + & + & + & - & + & + & - & - & 0 & 6 \\
\hline SRD & M & 13 & tronco & eutanásia & transicional & I & + & - & + & - & - & - & - & - & $1,6 \%$ & 0 \\
\hline Cocker & M & 13 & L4-L5 & - & transicional & I & + & - & + & - & $(+)$ & - & - & - & $7,6 \%$ & 8 \\
\hline Boxer & $\mathrm{F}$ & 9 & $\mathrm{C} 1-\mathrm{C} 2$ & - & transicional & I & + & - & + & - & - & - & - & - & 0 & 1 \\
\hline Spitz Alemão & $\mathrm{OF}$ & 8 & córtex & 600 dias & microcístico & I & $(+)$ & - & + & + & - & - & - & - & $7,2 \%$ & 0 \\
\hline Pitbull & M & 7 & córtex & - & papilar & I & + & - & + & - & $(+)$ & + & - & - & 0 & 2 \\
\hline Poodle & $\mathrm{F}$ & 13 & córtex & - & papilar & I & - & + & + & + & - & - & - & - & $0,5 \%$ & 1 \\
\hline
\end{tabular}

$\mathrm{SRD}=$ sem raça definida, $\mathrm{a}=$ presença de metástase, $\mathrm{M}=$ macho, $\mathrm{F}=$ fêmea, $\mathrm{C}=$ segmentos cervicais, $\mathrm{T}=$ segmentos torácicos. $\mathrm{L}=$ segmentos lombares. + positivo, - negativo. (+) fracamente positivo, VC = vermelho congo, PCK =anti-pancitoqueratina, GFAP = anti-proteína glial fibrilar ácida, Mit = número de mitoses em 10 campos de grande aumento. 
estão presentes comumente nos casos em que há acometimento da região infratentorial (mesencéfalo, ponte, bulbo e cerebelo) (Chrisman 1991, Bagley et al. 1999). A localização de meningiomas em córtex frontal resulta em síndrome cerebral, causando andar em círculos, convulsão e hipertensão intracraniana, que pode comprometer também o sistema vestibular (Braund 2003). Meningiomas são tumores cerebrais sem capacidade de gerar potenciais de ação, todavia $66 \%$ dos cães com meningioma cerebral apresentam convulsões (Schwartz et al. 2011). 0 crescimento lento de neoplasias menos infiltrativas pode isolar parcialmente regiões do parênquima cerebral, deixando-as mais excitáveis. A alta incidência de convulsões está relacionada a tumores que apresentam frequentemente displasia cortical ou outras anormalidades estruturais que estão associadas a propriedades epileptogênicas. Alterações morfológicas no tecido peritumoral, como a migração neuronal aberrante e alterações das vesículas sinápticas, também podem resultar nesse sinal clínico. A própria expansão do tecido tumoral por vezes leva a baixa perfusão tecidual e tumefação celular, que resultam em danos que aumentam a excitabilidade neuronal (van Breemen et al. 2007).

Já os animais com meningiomas espinhais apresentaram ataxia e sinais como déficit de propriocepção, paresia/ paraplegia ou tetraparesia/tetraplegia e alteração nos reflexos espinhais. Isto se deve ao acometimento das fibras ascendentes e descendentes da substância branca da medula espinhal. Independente do segmento medular afetado, a localização e a extensão da lesão é que determinarão a dificuldade locomotora do animal, podendo variar de monoparesia a tetraplegia (Braund 2003, Fernández \& Bernardini 2010).

Os exames para a visualização de massas e alterações no parênquima do SNC incluem em ordem de eficácia para o diagnóstico a mielografia, a tomografia computadorizada e a ressonância magnética (Kraft et al. 1997, Kraft \& Gavin 1999, Santos et al. 2012). Nossa instituição dispõe apenas de mielografia, o que permite a visualização de algumas alterações sugestivas de neoplasia espinhal. No caso de animais com alterações clínicas compatíveis com síndromes encefálicas, o diagnóstico definitivo só é realizado post mortem. Dos sete casos de neoplasia espinhal, a mielografia foi realizada em seis. Em um desses pacientes foi realizada a mielografia e devido ao não delineamento da lesão, o animal foi encaminhado para a realização de mielotomografia, que permitiu observar uma massa intradural-extramedular, o que é sugestivo de meningioma (Petersen et al. 2008). Nos outros cinco animais as alterações observadas foram compatíveis com lesão extradural em três, intradural-extramedular em um e intramedular em um. Nesse último paciente, na radiografia simples realizada previamente foi possível observar expansão do canal vertebral com alteração na lâmina dorsal do canal vertebral da vértebra L5. Em um paciente com lesão extradural, devido à inflitração da massa na vértebra $\mathrm{T} 1$, na radiografia foi observada diminuição do corpo da vértebra provavelmente por fratura patológica. Estas alterações ósseas são descritas em alguns casos de meningioma (Peterson et al. 2008).

Em cinco casos foram realizadas cirurgias para descom- pressão e/ou biopsia incisional. Nas neoplasias de região torácica (3) foram realizadas hemilaminectomias, na região cervical (1) e na vértebra L5 (1); laminectomias dorsais. Em dois dos pacientes observou-se que a massa era infiltrativa na medula espinhal, sendo realizada eutanásia no período transoperatório. Nos outros dois casos os proprietários não autorizaram o tratamento, optando pela eutanásia. A ocorrência de complicações trans e pós-operatórias foi descrita principalmente nos meningiomas cervicais, podendo resultar em óbito ou indicação de eutanásia (Asano et al. 2005). Nos quatro casos em que a suspeita clínica era de neoplasia em região intracraniana não foram realizados exames de ressonância magnética ou tomografia computadorizada, por não estarem disponíveis na instituição, além da impossibilidade do proprietário em realizar os exames nos locais indicados. Em um dos animais (Spitz Alemão) a suspeita inicial era meningencefalite linfocitária.

Nas neoplasias primárias do SNC a avaliação da sobrevida nos animais é limitada, dependendo de vários fatores. A maioria dos casos tem prognóstico reservado a mau (Braund 2003), influenciando na decisão imediata do proprietário pela eutanásia. A menor disponibilidade dos exames de imagens, seu custo e a limitação dos possíveis tratamentos são fatores que influenciam na decisão do proprietário e interferem nos dados de sobrevida destes animais. Todavia, segundo a literatura, a sobrevida de animais com meningiomas não infiltrativo tratados com cirurgia pode ser de quatro anos e quando há associação entre cirurgia e radioterapia pode chegar a seis anos (Petersen et al. 2008). As sequelas frequentemente observadas nos animais com meningiomas espinhais são incontinência urinária e fecal (Petersen et al. 2008).

As descrições macroscópicas observadas neste estudo são semelhantes à descrição prévia que refere nódulos firmes, aderidos à meninge que, por compressão, atrofiam o tecido nervoso adjacente (Braund \& Ribas 1986). Na avaliação histopatológica, os meningiomas foram subclassificados em transicional (4/11), meningotelial (2/11), papilar (2/11), angiomatoso (1/11), microcístico (1/11) e anaplásico (1/11). Os subtipos transicional e meningotelial são descritos como os mais frequentes em cães (Patnaik et al. 1986). Essa diversidade histológica é devida às origens embriológicas mistas das meninges, com células da crista neural e mesoderma (O’Rahilly \& Muller 1986). Todos os casos desse estudo estavam localizados perifericamente, com compressão e/ou invasão do parênquima nervoso adjacente.

Os meningiomas transicionais caracterizaram-se por uma mistura de padrão fibroso com meningotelial, com grupos de células sinciciais ou espirais concêntricas que formavam lóbulos bem demarcados, por vezes intercalados com células meningoteliais (Fig.1). Os meningiomas meningoteliais são descritos como um subtipo comum em cães, apesar de terem sido observados em apenas dois animais nesse estudo. Apresentam variação sincicial caracterizada por lóbulos de variados tamanhos, com tendência a espiral, delimitados por finos feixes de fibras colágenas, citoplasma abundante e não delimitado, núcleo ovoide e nucléolo evidente (Fig.2). Como observado na Figura 2, pode 
ocorrer formação de metaplasia óssea e cartilaginosa, mais comumente nos subtipos meningotelial e transicional. Nos meningiomas papilares observou-se formação de estrutura papilar ao redor de um centro vascular composto por células meningoteliais, com camadas de células meningo- teliais entremeadas (Fig.3) e ocasionalmente áreas de necrose multifocal. 0 meningioma angiomatoso apresentava numerosos vasos sanguíneos dilatados e hemorragia entre as células meningoteliais (Fig.4). 0 meningioma microcístico apresentava células fusiformes de núcleos alongados,
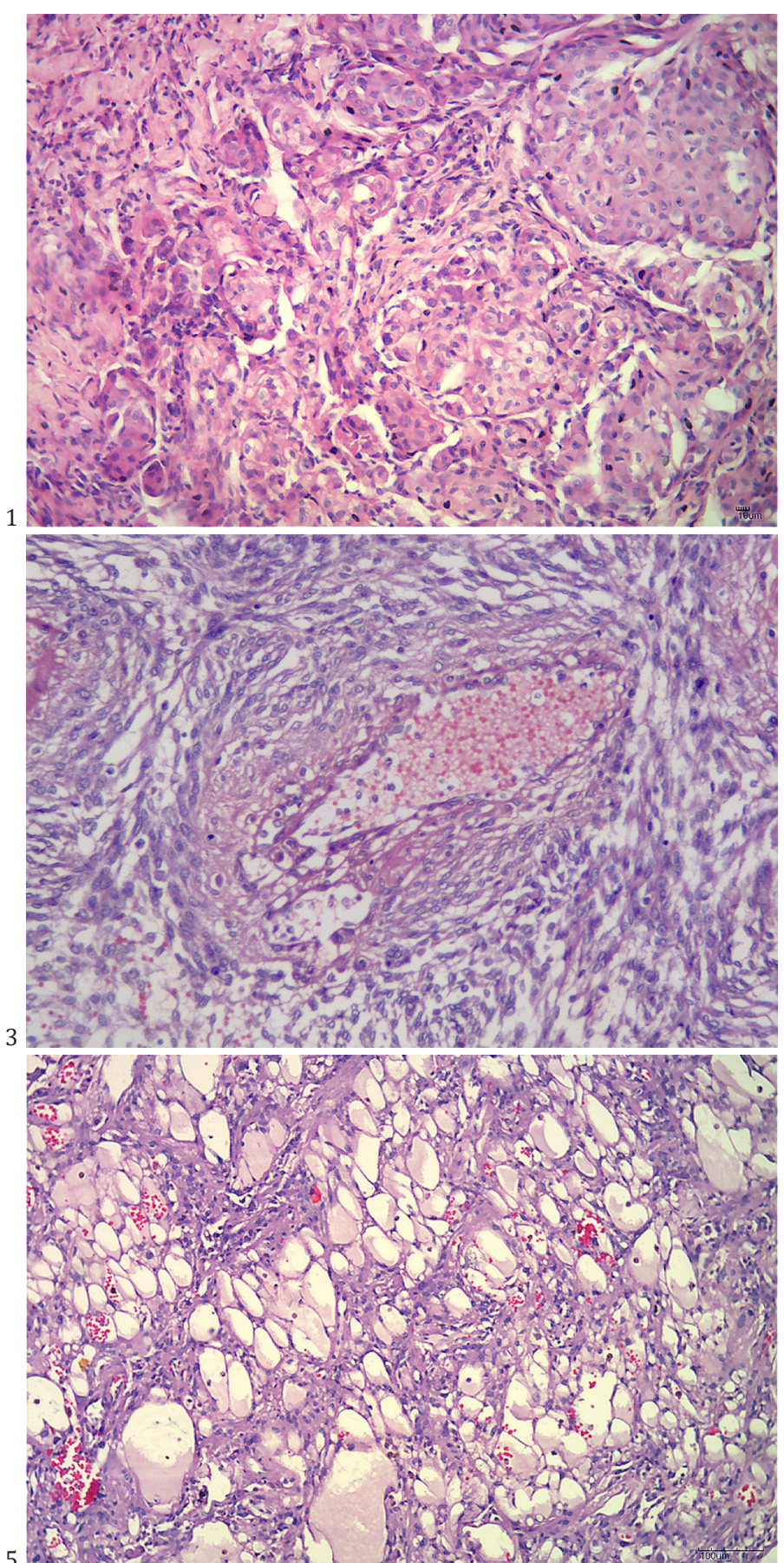

Fig.1. Meningioma transicional. Observam-se formações em espiral, com lóbulos bem demarcados. HE, obj.20x.

Fig.3. Meningioma papilar. Centro vascular com células meningoteliais ao redor formando estrutura de aspecto papilar. HE, obj.20x.

Fig.5. Meningioma microcístico. Observa-se grande quantidade de cistos bem delimitados, preenchidos por material amorfo discretamente basofílico. HE, obj.10x.
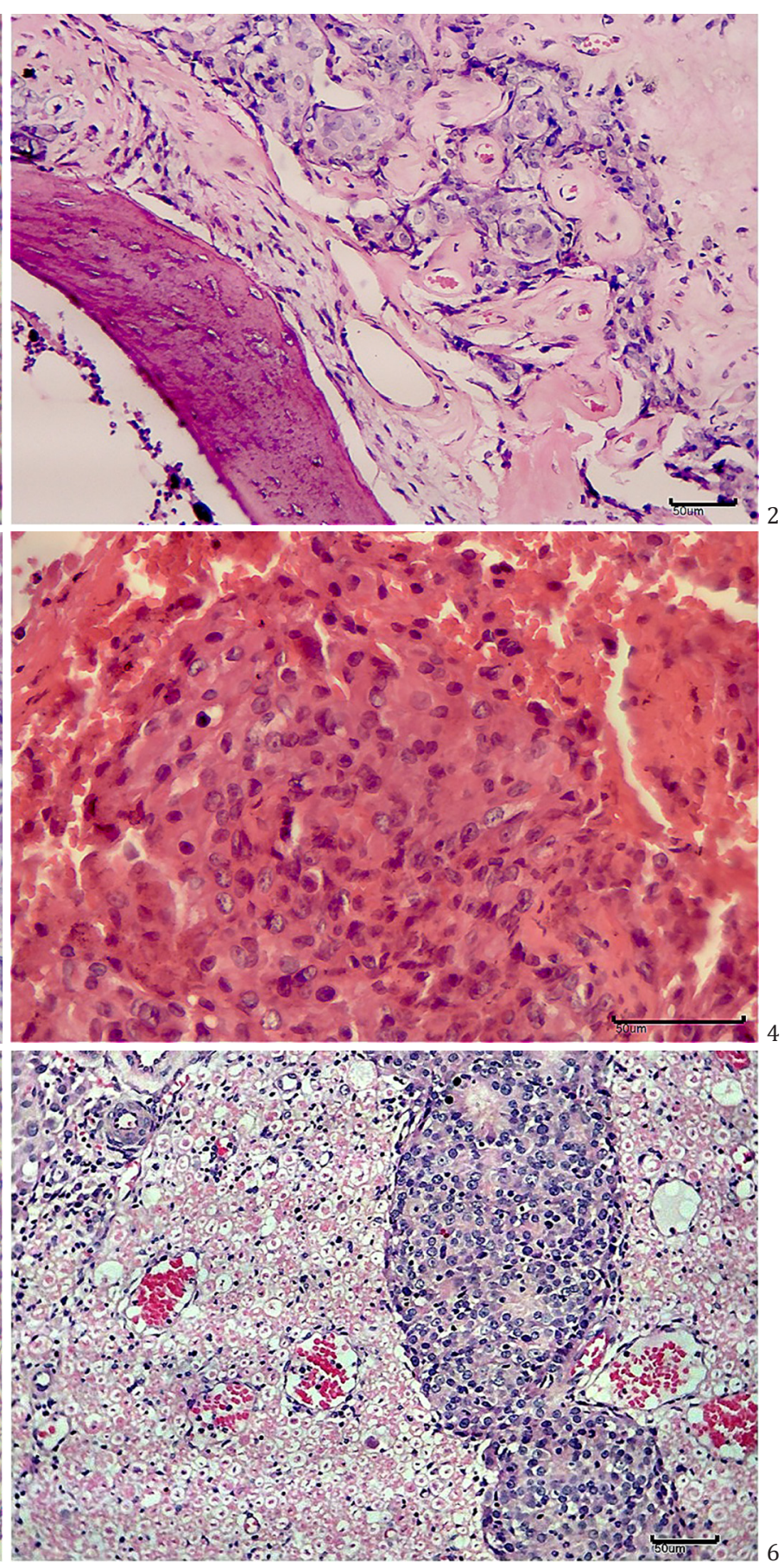

Fig.2. Meningioma meningotelial. Observam-se células neoplásicas dispostas em forma de ninhos adjacentes a metaplasia cartilaginosa. HE, obj.20x.

Fig.4. Meningioma angiomatoso. Observam-se hemorragia acentuada e no detalhe células neoplásicas com pleomorfismo moderado. HE, obj.20x. Detalhe, HE, obj.40x.

Fig.6. Meningioma anaplásico. Células neoplásicas com anisocariose moderada infiltradas entre gânglios nervosos. HE, obj.20x. 
de padrão frouxo, que formava cistos, contendo mucina ou não (Fig.5). 0 meningioma anaplásico (Fig.6) caracterizou-se por camadas sólidas de células meningoteliais com infiltração dos gânglios nervosos, áreas necróticas, pleomorfismo celular e alto índice mitótico (nove figuras de mitose/ campo grande aumento).

Os meningiomas apresentam forte coloração para colágeno e reticulina (Koestner \& Higgins 2002) que pode ser visualizada pela coloração de tricrômio de Masson (TM). Neste estudo foi constatada em $73 \%(8 / 11)$ dos casos (Quadro 2) intensa marcação para o TM entre as células neoplásicas (Fig.7). Estes resultados sugerem que a análise histoquímica com TM pode auxiliar no diagnóstico diferencial dos meningiomas.

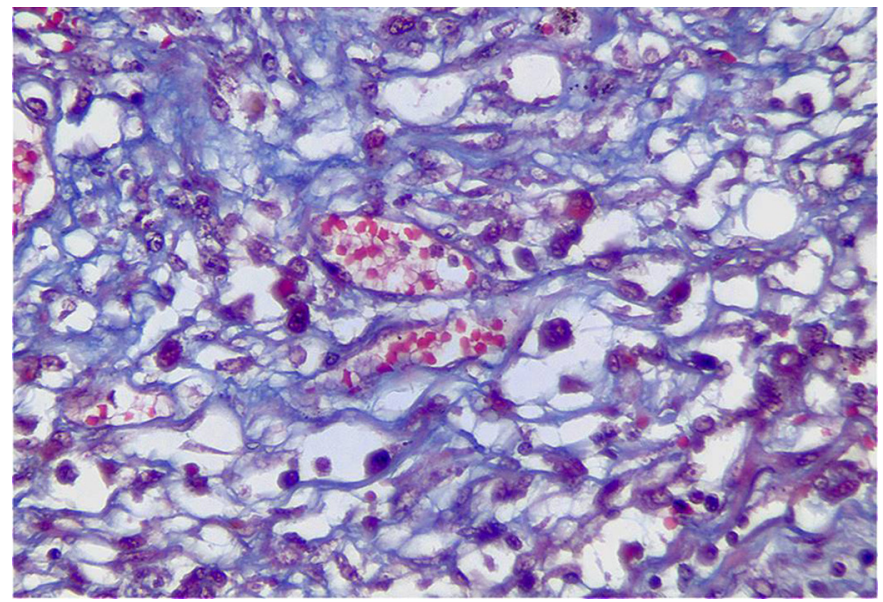

Fig.7. Meningioma anaplásico. São evidenciadas fibras colágenas, coradas em azul na coloração de tricrômio de Masson. TM, obj.40x.

$\mathrm{Na}$ análise dos cortes corados com vermelho congo foram observados pequenos depósitos de amiloide próximos às meninges em um caso. A presença de amiloide em meningiomas humanos é pouco frequente (Foschini et al. 1993) e em cães aparentemente está relacionado a casos atípicos (Schoniger et al. 2013).

0 painel imuno-histoquímico de acordo com o subtipo histológico está representado no Quadro 2. Em todos os casos verificou-se imunomarcação forte e difusa para vimentina, aspecto já relatado previamente em meningiomas (Barnhart et al. 2002, Montoliu et al. 2006). A imunomarcação para S100 foi de intensidade variável (6/11) e sempre menos intensa que a vimentina, apresentando um padrão inconsistente e com focos de células positivas. Os subtipos transicional e microcístico não apresentaram imunomarcação para GFAP em seis animais, no entanto, nos demais (5/11) houve marcação variável, o que difere de estudo prévio que relatou expressão positiva para GFAP em todos os casos de meningiomas fibroblásticos (Montoliu et al. 2006). Não se sabe se a expressão do GFAP nos meningiomas se deve à presença de astrócitos infiltrados ou se as células tumorais são positivas. Os subtipos microcístico, papilar e anaplásico apresentaram focos de marcação anti-pancitoqueratina fraca (menos de $30 \%$ das células marcadas) de modo semelhante ao relatado em estudo prévio (Barnhart et al. 2002).
A expressão do fator VIII foi negativa em todos os tumores (Quadro 2), contribuindo com o diagnóstico diferencial ao excluir tumores de origem vascular (Salvadori et al. 2011). Os resultados obtidos na análise imuno-histoquímica são semelhantes aos descritos em estudo prévio (Barnhart et al. 2002). No entanto, além dos anticorpos utilizados nesse estudo, há relatos em meningiomas de marcação variável para enolase neurônio específica (NSE) e negativa para sinaptofisina (Barnhart et al. 2002). A dupla positividade para vimentina e pancitoqueratina auxilia na diferenciação de meningioma das demais neoplasias do sistema nervoso (Barnhart et al. 2002).

Não se observou expressão de p53 no exame imuno-histoquímico em nenhum dos casos. Resultados similares foram relatados em cães com neoplasias intracranianas (Violin 2009), sugerindo que nos cães não há expressão de p53 nas células neoplásicas dos meningiomas. Alterações na expressão de p53 são observadas nos meningiomas em humanos (Pavelin et al. 2014), no entanto em animais há poucos dados sobre a expressão de p53 em neoplasmas (Koltai \& Vajdovich 2014).

A associação entre classificação histológica, localização e ocorrência ou não de metástase permite, de acordo com a Organização Mundial da Saúde, estabelecer a classificação dos meningiomas em graus de I a III (Koestner \& Higgins 2002, Louis et al. 2007). A graduação histológica e a avaliação da proliferação celular são utilizadas na avaliação do prognóstico. Neoplasias de grau I são consideradas de baixo potencial proliferativo e com possibilidade de cura seguida de ressecção cirúrgica (Louis et al. 2007). 0 meningioma anaplásico é graduado em grau III devido às características histopatológicas, como atipia nuclear e alto índice mitótico. A graduação I foi a mais frequente neste estudo (Quadro 2), assim como em outros relatos (Petersen et al. 2008). Em humanos, os menigiomas classificados como grau II (atípicos) e III (anaplásicos) são considerados desafiantes no diagnóstico e no tratamento (Fathi \& Roelcke 2013).

0 resultado da análise da proliferação celular está disposto no Quadro 2. 0 índice médio de proliferação celular foi de 3,4\% para a expressão de Ki-67 e de 3,2 figuras de mitose em 10 campos de grande aumento. A variação nos índices proliferativos (Ki-67) e, em alguns casos, ausência de marcação nos meningiomas já foi previamente descrita (Matiasek et al. 2009). Variações na intensidade de marcação da proteína Ki-67 podem também ocorrer dentro de um mesmo subtipo histológico (Isolan et al. 2005). De modo geral, os índices proliferativos nos meningiomas caninos, tanto em relação ao número de figuras de mitose (Schulman et al. 1992) como na expressão de Ki-67 são relativamente baixos, com índice médio de proliferação de 1,8\% (Matiasek et al. 2009). Outro aspecto interessante é que a baixa proliferação celular não está associada a um melhor prognóstico para o animal (Matiasek et al. 2009).

\section{CONCLUSÕES}

Os meningiomas foram o tipo histológico mais frequente dentre as neoplasias do SNC em cães, afetando animais adultos a idosos e principalmente machos. 
As técnicas histoquímicas utilizadas neste estudo contribuíram com a caracterização dos meningiomas e assim, na indisponibilidade do exame imuno-histoquímico, podem auxiliar na conclusão diagnóstica.

Apesar das variações na expressão de algumas proteínas, a imuno-histoquímica é o método disponível mais confiável para se estabelecer o diagnóstico de meningiomas, principalmente em casos atípicos.

Assim, a associação entre localização anatômica, caracterização histopatológica e imuno-histoquímica devem ser consideradas no diagnóstico de meningiomas. São necessários mais estudos em meningiomas em cães, que considerem a expressão de outros genes, de modo a contribuir com a determinação do prognóstico.

Agradecimentos.- À Fundação Araucária pelo auxílio financeiro (22954). Ao CNPq e CAPES pela concessão de bolsas de iniciação científica, de doutorado e produtividade em pesquisa.

\section{REFERÊNCIAS}

Adamo P.F., Cantile C. \& Steinberg H. 2003. Evaluation of progesterone and estrogen receptor expression in 15 meningiomas of dogs and cats. Am. J. Vet. Res. 64:1310-1318.

Asano K., Kadosawa T., Mori T., Miyamoto T., Ueno H., Watanabe K. \& Fujinaga T. 2005. Ventilatory failure and successful management for a dog with severe cervical meningioma. J. Vet. Med. Sci. 67:599-602.

Bagley R.S. 2010. Spinal neoplasms in small animals. Vet. Clin. North. Am., Small Anim. Pract. 40:915-927.

Bagley R.S., Gavin P.R., Moore M.P., Silver G.M., Harrington M.L. \& Connors R.L. 1999. Clinical signs associated with brain tumors in dogs: 97 cases (1992-1997). J. Am. Vet. Med. Assoc. 215:818-819.

Barnhart K.F., Wojcieszyn J. \& Storts R.W. 2002. Immunohistochemical staining patterns of canine meningiomas and correlation with published immunophenotypes. Vet. Pathol. 39:311-321.

Braund K.G. 2003. Braund's Clinical Neurology in Small Animals: localization, diagnosis and treatment. Neurological Syndromes, retrieved August 15, 2014, from <http://www.ivis.org/advances/Vite/braund1/ chapter_frm.asp?LA=1>

Braund K.G. \& Ribas J.L. 1986. Central nervous system meningiomas. Comped. Contin. Educ. Dent. 8:241-248.

Chrisman C.L. 1991. Head tilt, circling, nystagmus, and other vestibular deficits, p.269-296. In: Chrisman C.L. (Ed.), Problems in Small Animal Neurology. Lea and Febiger, Philadelphia.

Cordy D.R. 1978. Tumors of the nervous system, p.430-442. In: Moulton J.E. (Ed.), Tumors in Domestic Animals. The Regents of the University of California, California.

Davis F.G., McCarthy B.J., Freels S., Kupelian V. \& Bondy M.L. 1999. The conditional probability of survival of patients with primary malignant brain tumors: surveillance, epidemiology, and end results (SEER) data. Cancer 85:485-491.

Fathi A.R. \& Roelcke U. 2013. Meningioma. Curr. Neurol. Neurosci. Rep. 13:337.

Fernández V.L. \& Bernardini M. 2010. Neoplasias e síndromes paraneoplásicas, p.269-299. In: Fernández V.L. \& Bernardini M. (Eds), Neurologia em Cães e Gatos. MedVet, São Paulo. 450p.

Foschini M.P., D’Adda T., Bordi C. \& Eusebi V. 1993. Amyloid stroma in meningiomas. Virchows Arch. A Pathol. Anat. Histopathol. 422:53-59.

Frankhauser R., Luginbühl H. \& McGrath J.T. 1974. Tumours of the nervous system. Bull. World Health Organ. 50:53-69.

Hsu D.W., Efird J.T. \& Hedley-Whyte E.T. 1997. Progesterone and estrogen receptors in meningiomas: prognostic considerations. J. Neurosurg. 86:113-120.

Isolan G.R., Ribas Filho J.M., Isolan P.M., Giovanini A., Malafaia O., Dini L.I.,
Kummer Jr A., \& Negrao A.W. 2005. Neoplasias astrocitárias e correlação com as proteínas p53 mutada e Ki-67. Arq. Neuropsiquiatr. 63:9971004.

Jose-Lopez R., De la Fuente C., Pumarola M. \& Anor S. 2013. Spinal meningiomas in dogs: description of 8 cases including a novel radiological and histopathological presentation. Can. Vet. J. 54:948-954.

Koestner A. \& Higgins R. 2002. Tumors of the nervous system, p.697-738. In: Meuten D.J. (Ed.), Tumors in Domestic Animals. Iowa State Press, Iowa. 788p.

Koltai Z. \& Vajdovich P. 2014. Expression of multidrug resistance membrane transporter (Pgp) and p53 protein in canine mammary tumours. Acta Vet. Hung. 62:194-204.

Kraft S.L. \& Gavin P.R. 1999. Intracranial neoplasia. Clin. Tech. Small Anim. Pract. 14:112-123.

Kraft S.L., Gavin P.R., DeHaan C., Moore M., Wendling L.R. \& Leathers C.W. 1997. Retrospective review of 50 canine intracranial tumors evaluated by magnetic resonance imaging. J. Vet. Intern. Med. 11:218-225.

LeCouteur R.A. \& Withrow S.J. 2007. Tumors of nervous system, p.659685. In: Withrow S.J. \& Vail D.M. (Eds), Small Animal Clinical Oncology. Saunders Elsevier, Missouri.

Li Y.P., Zhang H.Z., She L., Wang X.D., Dong L., Xu E. \& Wang X.D. 2014. Primary extramedullary spinal melanoma mimicking spinal meningioma: a case report and literature review. Oncol. Lett. 8:339-344.

Long S. 2006. Neoplasia of the Nervous System. Retrieved August 15, 2014 from <http://www.ivis.org/advances/Vite/braund26/chapter. asp?LA=1>

Louis D.N., Ohgaki H., Wiestler O.D., Cavenee W.K., Burger P.C., Jouvet A., Scheithauer B.W. \& Kleihues P. 2007. The WHO classification of tumours of the central nervous system. Acta Neuropathol. 114:97-109.

Matiasek L.A., Platt S.R., Adams V., Scase T.J., Keys D., Miller J., Adamo F., Long S. \& Matiasek K. 2009. Ki-67 and vascular endothelial growth factor expression in intracranial meningiomas in dogs. J. Vet. Intern. Med. 23:146-151.

Montoliu P., Anor S., Vidal E. \& Pumarola M. 2006. Histological and immunohistochemical study of 30 cases of canine meningioma. J. Comp. Pathol. 135:200-207.

O’Rahilly R. \& Muller F. 1986. The meninges in human development. J. Neuropathol. Exp. Neurol. 45:588-608.

Patnaik A.K., Kay W.J. \& Hurvitz A.I. 1986. Intracranial meningioma: a comparative pathologic study of 28 dogs. Vet. Pathol. 23:369-373.

Pavelin S., Becic K., Forempoher G., Tomic S., Capkun V., Drmic-Hofman I., Mrklic I., Lusic I. \& Pogorelic Z. 2014. The significance of immunohistochemical expression of merlin, Ki-67, and p53 in meningiomas. Appl. Immunohistochem. Mol. Morphol. 22:46-49.

Petersen S.A., Sturges B.K., Dickinson P.J., Pollard R.E., Kass P.H., Kent M., Vernau K.M., Lecouteur R.A. \& Higgins R.J. 2008. Canine intraspinal meningiomas: imaging features, histopathologic classification, and long-term outcome in 34 dogs. J. Vet. Intern. Med. 22:946-953.

Platt S.R., Scase T.J., Adams V., Wieczorek L., Miller J., Adamo F. \& Long S. 2006. Vascular endothelial growth factor expression in canine intracranial meningiomas and association with patient survival. J. Vet. Intern. Med. 20:663-668.

Qiu L.H., Lui S., Zou L., Yue Q. \& Gong Q.Y. 2014. Lateral ventricular cystic meningioma: 2 rare case reports. Exp. Ther. Med. 7:1393-1395.

Salvadori C., Pintore M.D., Ricci E., Konar M., Tartarelli C.L., Gasparinetti N. \& Cantile C. 2011. Microcystic meningioma of the fourth ventricle in a dog. J. Vet. Med. Sci. 73:367-370.

Santos R.P., Fighera R.A., Beckmann D.V., Brum J.S., Ripplinger A., Neto D.P., Baumhardt R. \& Mazzanti A. 2012. Neoplasmas envolvendo o sistema nervoso central de cães: 26 casos (2003-2011). Pesq. Vet. Bras. 32:153158.

Schmitt F.C. 1999. Marcadores prognósticos em carcinoma mamário, p.3046. In: Alves V.A.F., Bacchi C.E. \& Vassallo J. (Eds), Manual de Imuno-histoquímica. Sociedade Brasileira de Patologia, São Paulo.

Scholzen T. \& Gerdes J. 2000. The Ki-67 protein: from the known and the unknown. J. Cell Physiol. 182:311-322. 
Schoniger S., Woolford L., Jutras L., Head E., De Lahunta A. \& Summers B. A. 2013. Unusual features in four canine meningiomas. J. Comp. Pathol. 149:237-241.

Schulman F.Y., Ribas J.L., Carpenter J.L., Sisson A.F. \& LeCouteur R.A. 1992. Intracranial meningioma with pulmonary metastasis in three dogs. Vet. Pathol. 29:196-202.

Schwartz M., Lamb C.R., Brodbelt D.C. \& Volk H.A. 2011. Canine intracranial neoplasia: clinical risk factors for development of epileptic seizures. J. Small Anim. Pract. 52:632-637.

Snyder J.M., Shofer F.S., Van Winkle T.J. \& Massicotte C. 2006. Canine intracranial primary neoplasia: 173 cases (1986-2003). J. Vet. Intern. Med. 20:669-675.

Stummer W. 2007. Mechanisms of tumor-related brain edema. Neurosurg. Focus 22:E8.

Summers B.A., Cummings J.F. \& De Lahunta A. 1995. Tumors of the central nervous system, p.351-401. In: Summers B.A., Cummings J.F. \&. De Lahunta A. (Eds), Veterinary Neuropathology. Mosby, Missouri. 527p.

Surawicz T.S., McCarthy B.J., Kupelian V., Jukich P.J., Bruner J.M. \& Davis F.G. 1999. Descriptive epidemiology of primary brain and CNS tumors: re- sults from the Central Brain Tumor Registry of the United States, 19901994. Neuro-Oncol. 1:14-25.

Tomek A., Cizinauskas S., Doherr M., Gandini G. \& Jaggy A. 2006. Intracranial neoplasia in 61 cats: localisation, tumour types and seizure patterns. J. Feline Med. Surg. 8:243-253.

Van Breemen M.S., Wilms E.B. \& Vecht C.J. 2007. Epilepsy in patients with brain tumours: epidemiology, mechanisms, and management. Lancet Neurol. 6:421-430.

Violin K.B. 2009. Neoplasias intracracianas em cães: avaliação imuno-histoquímica de marcadores de proliferação celular e expressão de p53. Dissertação de Mestrado em Patologia Experimental e Comparada, Faculdade de Medicina Veterinária e Zootecnia, Universidade de São Paulo, São Paulo, SP. 90p.

Wrensch M., Minn Y., Chew T., Bondy M. \& Berger M.S. 2002. Epidemiology of primary brain tumors: current concepts and review of the literature. Neuro-Oncol. 4:278-299.

Yu P.J., Hyman K.M., Cassiere H.A., Fallon B., Vatsia S.K., Esposito M.J. \& Glassman L.R. 2014. Metastatic meningioma extending into the left atrium through the pulmonary vein. Ann. Thorac. Surg. 97:2173-2175. 